\title{
Planetary Systems Dynamics Eccentric patterns in debris disks \& Planetary migration in binary systems
}

\author{
V. Faramaz ${ }^{1}$, H. Beust ${ }^{1}$, J.-C. Augereau ${ }^{1}$, A. Bonsor ${ }^{1}$, P. Thébault ${ }^{2}$, \\ Y. $\mathbf{W u}^{3}$, J. P. Marshall ${ }^{4}$, C. del Burgo ${ }^{5}$, S. Ertel ${ }^{1}$, C. Eiroa $^{4}$, \\ B. Montesinos ${ }^{6}$, A. Mora ${ }^{7}$, and the DUNES team \\ ${ }^{1}$ UJF-Grenoble 1 / CNRS-INSU, Institut de Planétologie et d'Astrophysique \\ de Grenoble (IPAG) UMR 5274, Grenoble, F-38041, France \\ ${ }^{2}$ LESIA, Observatoire de Paris, 92195 Meudon France \\ ${ }^{3}$ Dpt. of Astronomy and Astrophysics, University of Toronto, ON M5S 3H4, Canada \\ ${ }^{4}$ Dpt. Fìsica Teòrica, Facultad de Ciencias, Universidad Autònoma \\ de Madrid, Cantoblanco, 28049 Madrid, Spain \\ ${ }^{5}$ Instituto Nacional de Astrofísica, Óptica y Electrónica, \\ Luis Enrique Erro 1, Sta. Ma. Tonantzintla, Puebla, Mexico \\ ${ }^{6}$ Dpt. de Astrofisica, Centro de Astrobiologìa (INTA-CSIC), ESAC Campus, \\ P.O.Box 78, E-28691 Villanueva de la Cañada, Madrid, Spain \\ ${ }^{7}$ Aurora Technology B.V., ESA-ESAC Gaia SOC. P.O. Box 78, \\ E28691 Villanueva de la Cañada, Madrid, Spain
}

\begin{abstract}
We present some highlights of two ongoing investigations that deal with the dynamics of planetary systems. Firstly, until recently, observed eccentric patterns in debris disks were found in young systems. However recent observations of Gyr-old eccentric debris disks leads to question the survival timescale of this type of asymmetry. One such disk was recently observed in the far-IR by the Herschel Space Observatory around $\zeta^{2}$ Reticuli. Secondly, as a binary companion orbits a circumprimary disk, it creates regions where planet formation is strongly handicapped. However, some planets were detected in this zone in tight binary systems ( $\gamma$ Cep, HD 196885). We aim to determine whether a binary companion can affect migration such that planets are brought in these regions and focus in particular on the planetesimal-driven migration mechanism.
\end{abstract}

Keywords. Circumstellar matter - Methods: N-body Simulations - Celestial mechanics - Stars: $\zeta^{2}$ Reticuli, planetary systems

Can eccentric debris disks be long-lived? Recent Herschel far-IR observations of the debris disk surrounding the 2-3 Gyr old star $\zeta^{2}$ Reticuli, obtained as part of the DUNES key program (see Eiroa et al., 2013), reveal an asymmetric double-lobed circumstellar feature (Fig. 1, left panel), interpreted as a ring like structure seen almost edge-on with an elliptical shape and minimum eccentricity of 0.3, extending from 70 to $120 \mathrm{AU}$ (Eiroa et al., 2010). So far, eccentric patterns in debris disks were observed in young systems (HR 4796: 10 Myr ; Fomalhaut: $440 \mathrm{Myr}$ ), and attributed to the dynamical shaping of the disk by massive companion on an eccentric orbit (see e.g. Wyatt, 1999). We discuss here the origin of this eccentric pattern in the $\zeta^{2}$ Reticuli system, and the ability to maintain the disk asymmetry over Gyrs. Through a modeling method combining analytical predictions, numerical N-body treatment (Levison \& Duncan, 1994) and radiative transfer, we can put constraints on the orbital parameters of hypothetical perturbers that could induce a disk structure compatible with observational constraints. The synthetic images support the hypothesis that the observed double-lobe feature is the signature of an eccentric ring (see Fig. 1, right panel), and our synthetic model supports the presence 

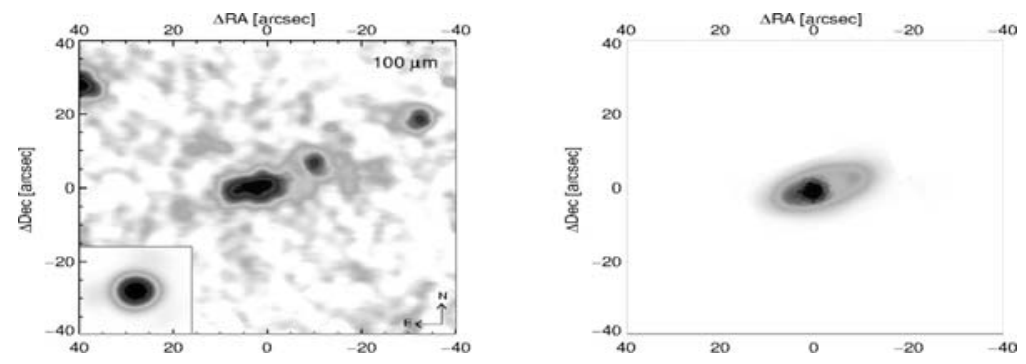

Figure 1. Left: Herschel/PACS Observations of $\zeta^{2}$ Reticuli at 100 microns. Right: Synthetic image at 100 microns.

of a planetary mass companion orbiting at several tens of AU from its host star, and on a significantly eccentric orbit (Faramaz et al. 2013, in prep.).

Planetary migration in binary systems Planetesimal-driven migration mechanism leads a planet to exchange angular momentum with planetesimals during close encounters. Angular momentum passes from outer to inner material and components experience a mutual gravitational attraction. In a system with a single planet, timescales favour interactions with inner material, and thus inward migration (see Fig. 1 of Kirsh et al. 2009). Our goal is to investigate this process in binary systems: preliminary results show that in the presence of a binary companion, the direction of migration could be reversed: interactions with outer material seems favoured, which would move planets out rather than in (see Fig. 2). This may provide an explanation for the presence of planets in regions of binary systems where models fail to explain in situ formation (see the review by Thébault P., 2011).

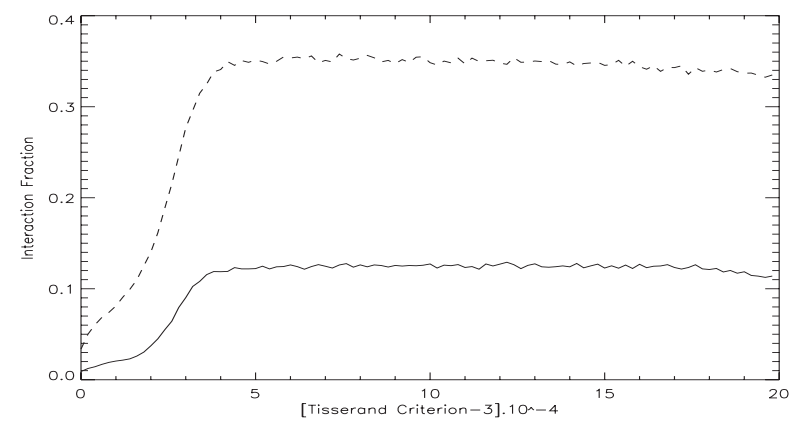

Figure 2. Probability for a planetesimal to suffer a close encounter as a function of its Tisserand parameter, and thus as a function of its distance to a planet of mass $4.5 M_{\oplus}$ at $10 \mathrm{AU}$ on a circular orbit in the presence of a $1 M_{\odot}$ binary companion on a circular orbit at $100 \mathrm{AU}$, within $3 \mathrm{R}_{\mathrm{Hill}}$, and for inner (solid line) and outer (dashed line) material.

\section{References}

Eiroa, C., et al. 2010, A\&A, 518, L131

Eiroa, C., et al. 2013, A\&A, 555, A11

Faramaz, V., et al., In prep.

Kirsh, D. R., Duncan, M., Brasser, R., \& Levison, H. F. 2009, Icarus, 199, 197-209

Levison, H. F. \& Duncan, M. J 1994, Icarus, vol. 108, no. 1, p. 18-36

Thébault, P. 2011, Celestial Mechanics and Dynamical Astronomy, vol. 111, p. 29-49

Wyatt, M. C. 1999, ApJ, 527:918-944

Computations presented in this paper were performed at the Service Commun de Calcul Intensif de l'Observatoire de Grenoble (SCCI) on the super-computer funded by Agence Nationale pour la Recherche under contracts ANR-07-BLAN-0221, ANR-2010-

Funding for this project was provided by a grant from la Région Rhône-Alpes.

C. Eiroa, J.P. Marshall, and B. Montesinos are supported partly by Spanish grant AYA 2011-26202. 